\title{
Pulmonary artery enlargement and mortality risk in moderate to severe COPD: results from COPDGene
}

\author{
To the Editor:
}

COPD is one of the leading causes of mortality worldwide [1]. Pulmonary hypertension has been associated with reduced survival among individuals with COPD [2] and is an independent risk factor for mortality following acute exacerbations of COPD (AECOPD) [3]. Measurement of the pulmonary artery to aorta (PA:A) ratio by computed tomography (CT) and assessment of pulmonary artery enlargement (PA:A >1) outperforms echocardiography in identifying pulmonary hypertension in severe COPD [4], and pulmonary artery enlargement has been independently associated with risk for total and severe AECOPD in two large prospective COPD cohorts [5]. Studies of population-based [6] and non-COPD [7] cohorts have indicated an association between pulmonary artery enlargement on CT and mortality. However, these studies did not include adjustment for other factors associated with COPD severity, known risk factors for COPD mortality and risk factors for atherosclerotic cardiovascular disease (ASCVD), which is a major cause of mortality in COPD.

We analysed CT imaging and clinical data from the COPDGene study and hypothesised that pulmonary artery enlargement is associated with all-cause mortality, even after adjustment for other known predictors of mortality in COPD and risk factors for ASCVD. We analysed data from 3464 COPDGene participants with Global Initiative for Chronic Obstructive Lung Disease spirometry grades 2-4 COPD [5] who had CT imaging, and vital status assessment $\sim 6$ years later. This cohort underwent detailed baseline assessment [8], volumetric non-contrast CT scans of the chest with measurement of pulmonary artery and aorta at the level of the pulmonary artery bifurcation [5], and long-term longitudinal clinical follow-up [9], as described previously. All-cause mortality was determined via longitudinal follow-up, contact with next-of-kin, clinical records, death certificates and information from the Social Security Death Index [10].

Participants had a median follow-up period of 6.3 years (interquartile range 1.7 years, range 8.4 years). Mean \pm SD age was $63 \pm 9$ years; $44 \%$ were female and $78 \%$ were white. The mean forced expiratory volume in $1 \mathrm{~s}\left(\mathrm{FEV}_{1}\right)$ was $50 \pm 18 \%$ predicted, and $28 \%$ of participants used supplemental oxygen. Mean PA:A was $0.90 \pm 0.14$ and 810 (23.4\%) participants had PA:A >1. During the follow-up period, 755 (21.8\%) participants died. In Kaplan-Meier analysis (SPSS, IBM, Armonk, NY, USA), participants with PA:A >1 had shorter mean estimated survival (6.7 (95\% CI 6.50-6.89) years versus 7.4 (95\% CI 7.35-7.53) years; $\mathrm{p}<0.001$ (log-rank); figure 1).

Cox proportional hazards $(\mathrm{CPH})$ models were used to investigate for potential association between pulmonary artery enlargement (PA:A >1) and mortality. The multivariable $\mathrm{CPH}$ model included covariates that adjusted for other known factors associated with mortality and disease severity in COPD, as well as ASCVD. These included demographics (age, sex, race), body mass index (BMI), use of supplemental oxygen, 6-min walk distance (6MWD), modified Medical Research Council (mMRC) dyspnoea score, $\mathrm{FEV}_{1}$ (\% predicted), history of severe AECOPD within the preceding 12 months (self-reported using questions modified from standardised questionnaires), percentage emphysema on CT (Slicer software; www.slicer.org), current smoking and self-reported histories of coronary artery disease, diabetes mellitus, hypertension and hyperlipidaemia. In the unadjusted $\mathrm{CPH}$ model, pulmonary artery enlargement was associated with hazard ratio (HR) for mortality of 1.75 (95\% CI $1.50-2.04 ; \mathrm{p}<0.001$ ). In

@ERSpublications

Pulmonary artery enlargement on chest CT imaging is independently associated with all-cause mortality in moderate-severe COPD, after adjustment for other known risk factors for COPD mortality and cardiovascular disease http://bit.ly/2rdYOR1

Cite this article as: LaFon DC, Bhatt SP, Labaki WW, et al. Pulmonary artery enlargement and mortality risk in moderate to severe COPD: results from COPDGene. Eur Respir J 2020; 55: 1901812 [https://doi. org/10.1183/13993003.01812-2019]. 
FIGURE 1 Association between pulmonary artery enlargement and all-cause mortality in COPD. KaplanMeier survival curves stratified by the presence or absence of pulmonary artery enlargement $(p<0.001$, logrank test). PA:A: pulmonary artery to aorta ratio.

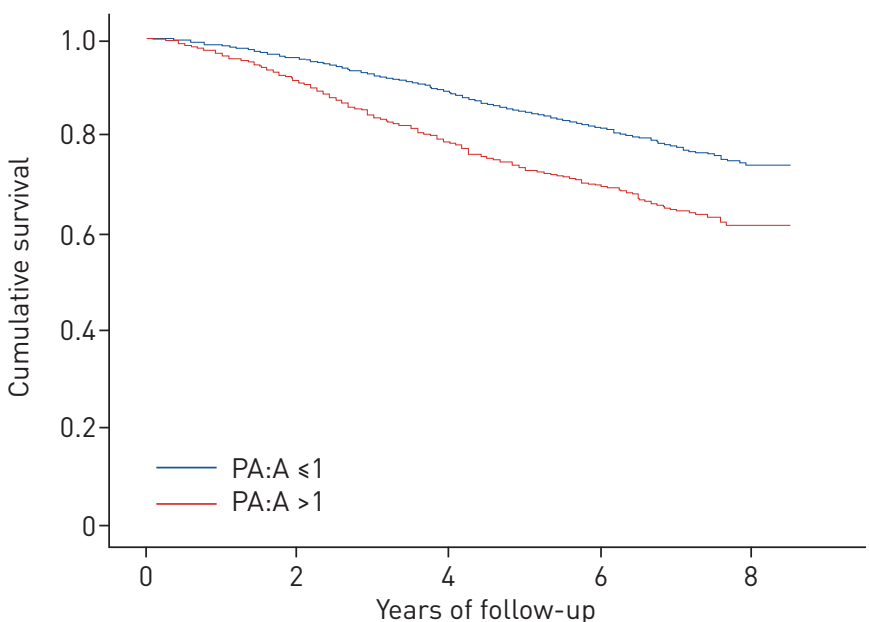

the multivariable $\mathrm{CPH}$ model, pulmonary artery enlargement was associated with a $42 \%$ increase in mortality risk (HR 1.42, 95\% CI 1.17-1.72; p<0.001). Older age, male sex, lower BMI, lower FEV $(\%$ pred), shorter 6MWD, supplemental oxygen use, higher mMRC dyspnoea scores and current smoking were also associated with increased mortality risk in the adjusted CPH model. ASCVD-specific factors were not associated with risk for mortality in this cohort.

Our results are the first to demonstrate an association between pulmonary artery enlargement on CT and mortality in moderate to severe COPD, even when adjusted for factors including AECOPD, components of the BODE (BMI, airflow obstruction, dyspnoea, exercise capacity) index and ASCVD. Several studies have previously reported associations between CT-based PA:A ratio and mortality. Using coronary CT angiography from patients with suspected coronary artery disease, NAKANISHI et al. [7] demonstrated an association between pulmonary artery enlargement (defined as PA:A $>0.9$ in that study) and mortality after adjustment for cardiovascular risk factors and left ventricular function. However, this study neither reported the prevalence of chronic lung disease nor performed adjustment for factors associated with it. ASCVD is a major cause of mortality in COPD, and several factors such as age, sex and smoking are related to both ASCVD risk and COPD severity/mortality. However, the results of our adjusted model suggest that the increased risk is unlikely to be attributable to ASCVD.

SHIN et al. [11] found an association between PA:A $\geqslant 1$ and mortality over a shorter follow-up interval, in a smaller cohort of COPD patients referred for lung transplant/lung volume reduction evaluation, after adjustment for age, BMI and $\mathrm{FEV}_{1}$ (\% pred). Additionally, TERZIKHAN et al. [6] reported an independent association between PA:A ratio and mortality among participants with COPD in a large, predominantly white population in the Netherlands. However, only $5 \%$ of the study population had moderate-severe COPD, and 17 out of the total 2197 participants had PA:A $\geqslant 1$. In contrast to prior studies, COPDGene is a multicentre, disease-specific study that included larger numbers of African-American participants (approximately one-third of total enrolment) [8] as well large numbers of subjects with moderate to severe disease. In addition, our results indicate a high prevalence of pulmonary artery enlargement in the study cohort, with pulmonary artery enlargement in nearly a quarter of participants. Our findings add to the mounting body of evidence to indicate an association between PA:A ratio and adverse outcomes in COPD including severe acute exacerbations [5], reduced exercise tolerance [12] and risk of development of resting hypoxaemia [13] as well as cardiac injury and more complicated hospital course during severe acute exacerbations [14].

Our results demonstrate increased mortality risk after adjustment for supplemental oxygen use, $\mathrm{FEV}_{1}$ and percentage emphysema. This suggests that COPD severity cannot completely account for the observed associations, and supports the importance of mechanisms other than chronic hypoxia and capillary dropout due to emphysema in the pathophysiology of pulmonary vascular disease in COPD. Direct pulmonary vascular injury as a result of cigarette smoke exposure could also contribute to the development of pulmonary hypertension in early or mild disease, in the absence of emphysema or hypoxaemia [15]. Genetic variants in IREB2 and GALC that were identified through a genome-wide association study of COPDGene and ECLIPSE (Evaluation of COPD Longitudinally to Identify Predictive Surrogate End-points) participants with PA:A >1 [16] might also account for earlier onset of pulmonary hypertension in certain individuals. 
The strengths of our study include use of a well-defined cohort, length of follow-up and adjustment for other known predictors of mortality. PA:A ratio measurement represents a reproducible, practical method for evaluation of patients with COPD for pulmonary vascular disease. Our study has several limitations. First, our results should not be used to imply a direct causal relationship between pulmonary artery enlargement and mortality. Since we evaluated all-cause mortality, there is insufficient evidence to reach conclusions regarding specific causes of death among individuals with concurrent COPD and pulmonary artery enlargement. Measurement of disease-specific outcomes and study of PA:A ratio in common comorbid conditions such as obesity, obstructive sleep apnoea and congestive heart failure would be valuable in elucidating the nature of the associations observed in this study. Furthermore, our conclusions are based on a single cohort with moderate to severe disease, using non-contrast, non-gated CT imaging, though others have reached similar conclusions using different study populations and CT imaging protocols $[6,7,17]$.

These findings extend the known utility of pulmonary artery enlargement on CT scan as an imaging-based biomarker for morbidity and mortality in COPD. The increased use of CT imaging for clinical indications (i.e. lung cancer screening) coupled with the relative ease in ascertaining pulmonary artery enlargement make this a practical metric for aiding in COPD prognosis.

David C. LaFon $\oplus^{1,2}$, Surya P. Bhatt ${ }^{1,2,3}$, Wassim W. Labaki $\oplus^{4}$, Farbod N. Rahaghi ${ }^{5}$, Matthew Moll ${ }^{5,6}$, Russ P. Bowler ${ }^{7}$, Elizabeth A. Regan $\oplus^{8}$, Barry J. Make $\odot^{7}$, James D. Crapo ${ }^{7}$, Raul San Jose Estepar ${ }^{9}$, Alejandro A. Diaz ${ }^{5}$, Edwin K. Silverman ${ }^{5,6}$, MeiLan K. Han ${ }^{4}$ Brian Hobbs $\oplus^{5,6}$, Michael H. Cho $\oplus^{5}$, George R. Washko ${ }^{5}$, Mark T. Dransfield ${ }^{1,2,10}$ and J. Michael Wells ${ }^{1,2,10}$ for the COPDGene Investigators ${ }^{11}$

${ }^{1}$ Division of Pulmonary, Allergy, and Critical Care Medicine, University of Alabama at Birmingham (UAB), Birmingham, AL, USA. ${ }^{2} \mathrm{UAB}$ Lung Health Center, Birmingham, AL, USA. ${ }^{3}$ UAB Lung Imaging Core, Birmingham, AL, USA. ${ }^{4}$ Division of Pulmonary and Critical Care Medicine, University of Michigan, Ann Arbor, MI, USA. ${ }^{5}$ Division of Pulmonary and Critical Care Medicine, Brigham and Women's Hospital, Harvard Medical School, Boston, MA, USA. ${ }^{6}$ Channing Division of Network Medicine, Brigham and Women's Hospital, Harvard Medical School, Boston, MA, USA. ${ }^{7}$ Division of Pulmonary and Critical Care Medicine, National Jewish Health, Denver, CO, USA. ${ }^{8}$ Division of Rheumatology, Dept of Medicine, National Jewish Health, Denver, CO, USA. ${ }^{9}$ Applied Chest Imaging Laboratory, Dept of Radiology, Brigham and Women's Hospital, Harvard Medical School, Boston, MA, USA. ${ }^{10}$ Birmingham VA Medical Center, Birmingham, AL, USA. ${ }^{11} \mathrm{~A}$ complete list of COPDGene Investigators is available at COPDGene.org.

Correspondence: David C. LaFon, Division of Pulmonary, Allergy, and Critical Care Medicine, University of Alabama at Birmingham, THT422, 1900 University Blvd, Birmingham, AL, USA 35294. E-mail: dlafon@uabmc.edu

Received: 06 Sept 2019 | Accepted after revision: 23 Oct 2019

Acknowledgments: The authors acknowledge the COPDGene Core Teams and COPDGene Investigators. A complete list of these personnel is available at COPDGene.org.

Author contributions: D.C. LaFon, M.T. Dransfield and J.M. Wells all provided substantial contributions to the conception and design of the work as well as the acquisition, analysis and interpretation of data for the work. D.C. LaFon, S.P. Bhatt, M.T. Dransfield and J.M. Wells drafted the work and all authors revised the manuscript critically for important intellectual content and gave final approval of the version to be published. D.C. LaFon and J.M. Wells agree to be accountable for all aspects of the work in ensuring that questions related to the accuracy or integrity of any part of the work are appropriately investigated and resolved.

Support statement: This work was supported by grants from the National Institutes of Health (NIH): 5T32HL105346-9 (to D.C. LaFon), K08HL123940 (to J.M. Wells). The Genetic Epidemiology of COPD Program is supported by National Institutes of Health Grants U01 HL089897 and U01 HL089856. Funding information for this article has been deposited with the Crossref Funder Registry.

Conflict of interest: D.C. LaFon reports grants from NIH (5T32HL105346-9), during the conduct of the study. S.P. Bhatt reports grants from NIH (K23HL133438), during the conduct of the study; contract for research from ProterixBio, personal fees for advisory board work from Sunovion and GlaxoSmithKline, outside the submitted work. W.W. Labaki has nothing to disclose. F.N. Rahaghi has nothing to disclose. M. Moll has nothing to disclose. R.P. Bowler has nothing to disclose. E.A. Regan has nothing to disclose. B.J. Make reports funding from the NHLBI for the COPDGene study; grants and medical advisory board work from Boehringer Ingelheim, GlaxoSmithKline, AstraZeneca and Sunovian; personal fees for data monitoring board work from Spiration and Shire/Baxalta; CME personal fees from WebMD, National Jewish Health, American College of Chest Physicians, Projects in Knowledge, Hybrid Communications, SPIRE Learning, Ultimate Medical Academy, Catamount Medical, Eastern Pulmonary Society, Catamount Medical Communications Medscape, Eastern VA Medical Center, Academy Continued Healthcare Learning, and Mt. Sinai Medical Center; royalties from Up-To-Date; medical advisory board work from Novartis, Phillips, Third Pole, Science 24/7 and Vernoa; grants from Pearl; outside the submitted work. J.D. Crapo has nothing to disclose. R. San Jose Estepar reports grants from NIH/NHLBI, during the conduct of the study; grants from NIH/NHLBI, personal fees from Toshiba, Boehringer Ingelheim and Eolo Medical, outside the submitted work; and is a founder and co-owner of Quantitative Imaging Solutions, which is a company that provides image based consulting and develops software to enable data sharing. A.A. Diaz reports grants from National Institutes of Health (R01-HL133137), Minority Faculty Development Career Award from Brigham and Women's Hospital, during the conduct of the study. E.K. Silverman reports grants from $\mathrm{NIH}$, during the conduct of the study; grants and travel support from GlaxoSmithKline, outside the 
submitted work. M.K. Han reports grants from NIH, during the conduct of the study; personal fees from GSK, BI, Mylan and AstraZeneca, research support from Novartis and Sunovion, outside the submitted work. B. Hobbs has nothing to disclose. M.H. Cho reports grants from NIH, during the conduct of the study; grants from GSK, personal fees from Genentech, outside the submitted work. G.R. Washko has nothing to disclose. M.T. Dransfield reports grants from NIH, during the conduct of the study; grants from Department of Defense, personal fees for consultancy from and contracted clinical trials for Boehringer Ingelheim, GlaxoSmithKline, AstraZeneca, Boston Scientific and PneumRx/BTG, contracted clinical trials for Novartis, Yungin and Pulmonx, personal fees for consultancy from Genentech, Quark Pharmaceuticals and Mereo, grants from NIH and American Lung Association, outside the submitted work. J.M. Wells reports grants from NIH/NHLBI (K08 HL123940), during the conduct of the study; grants from NIH/NCATS (UH3 TR002450) and Bayer, grants and advisory board work from GSK and Mereo BioPharma, advisory board work from Boehringer Ingelheim, endpoint adjudication from Quintiles and PRA, outside the submitted work.

\section{References}

1 Burney PG, Patel J, Newson R, et al. Global and regional trends in COPD mortality, 1990-2010. Eur Respir J 2015; 45: 1239-1247.

2 Weitzenblum E, Hirth C, Ducolone A, et al. Prognostic value of pulmonary artery pressure in chronic obstructive pulmonary disease. Thorax 1981; 36: 752-758.

3 McGhan R, Radcliff T, Fish R, et al. Predictors of rehospitalization and death after a severe exacerbation of COPD. Chest 2007; 132: 1748-1755.

4 Iyer AS, Wells JM, Vishin S, et al. CT scan-measured pulmonary artery to aorta ratio and echocardiography for detecting pulmonary hypertension in severe COPD. Chest 2014; 145: 824-832.

5 Wells JM, Washko GR, Han MK, et al. Pulmonary arterial enlargement and acute exacerbations of COPD. N Engl J Med 2012; 367: 913-921.

6 Terzikhan N, Bos D, Lahousse L, et al. Pulmonary artery to aorta ratio and risk of all-cause mortality in the general population: the Rotterdam Study. Eur Respir J 2017; 49: 1602168.

7 Nakanishi R, Rana JS, Shalev A, et al. Mortality risk as a function of the ratio of pulmonary trunk to ascending aorta diameter in patients with suspected coronary artery disease. Am J Cardiol 2013; 111: 1259-1263.

8 Regan EA, Hokanson JE, Murphy JR, et al. Genetic epidemiology of COPD (COPDGene) study design. COPD 2010; 7: 32-43.

9 Stewart JI, Moyle S, Criner GJ, et al. Automated telecommunication to obtain longitudinal follow-up in a multicenter cross-sectional COPD study. COPD 2012; 9: 466-472.

10 Criner RN, Labaki WW, Regan EA, et al. Mortality and exacerbations by Global Initiative for Chronic Obstructive Lung Disease groups ABCD: 2011 versus 2017 in the COPDGene cohort. Chronic Obstr Pulm Dis 2019; 6: 64-73.

11 Shin S, King CS, Brown AW, et al. Pulmonary artery size as a predictor of pulmonary hypertension and outcomes in patients with chronic obstructive pulmonary disease. Respir Med 2014; 108: 1626-1632.

12 Wells JM, Iyer AS, Rahaghi FN, et al. Pulmonary artery enlargement is associated with right ventricular dysfunction and loss of blood volume in small pulmonary vessels in chronic obstructive pulmonary disease. Circ Cardiovasc Imaging 2015; 8: e002546.

13 Wells JM, Estepar RS, McDonald MN, et al. Clinical, physiologic, and radiographic factors contributing to development of hypoxemia in moderate to severe COPD: a cohort study. BMC Pulm Med 2016; 16: 169.

14 Wells JM, Morrison JB, Bhatt SP, et al. Pulmonary artery enlargement is associated with cardiac injury during severe exacerbations of COPD. Chest 2016; 149: 1197-1204.

15 Voelkel NF, Gomez-Arroyo J, Mizuno S. COPD/emphysema: the vascular story. Pulm Circ 2011; 1: 320-326.

16 Lee JH, Cho MH, Hersh CP, et al. IREB2 and GALC are associated with pulmonary artery enlargement in chronic obstructive pulmonary disease. Am J Respir Cell Mol Biol 2015; 52: 365-376.

17 de-Torres JP, Ezponda A, Alcaide AB, et al. Pulmonary arterial enlargement predicts long-term survival in COPD patients. PLoS One 2018; 13: e0195640. 